\title{
Wear of elastic tube with nonuniform coating by rigid bush
}

\author{
Kirill E Kazakov,2,* \\ ${ }^{1}$ Ishlinsky Institute for Problems in Mechanics RAS, 119526, pr-t Vernadskogo 101-1, Moscow, Russia \\ ${ }^{2}$ Bauman Moscow State Technical University, 105005, 2ya Baumanskaya 5-1, Moscow, Russia
}

\begin{abstract}
This article is devoted to the statement and construction of analytical solution of the wearcontact problem for a rigid bush and elastic pipe with a coating in the case when the coating is nonuniform. The presence of nonuniformity leads us to the necessity of constructing a solution in a special form over special functions, since standard methods does not allow us to effectively take into account the complex properties of the coating. Analytical representation for contact stresses under the bush is presented in series with separate factor, which connect with complex properties of coating. This allows provide effective calculation even if these properties are described by rapidly changing or discontinuous functions. It is also shown that contact stresses will be negligible over time.
\end{abstract}

\section{Introduction}

Analytical solutions of model contact and wear-contact problems is extremely important for testing numerical algorithms for solving more complex problems. Such problems are test for numerical algorithms. One of the problems whose solution can be constructed analytically is considered in this article.

Earlier, wear-contact problems for bodies with complex coatings in plane and axisymmetric formulations were considered [1-3]. In these works, contact and wear-contact problems for bodies with similar coatings were investigated, but problems for cylindrical bodies were not considered. This work is devoted to the formulation and solution of the cylindrical wear-contact problem. For solution constructing we use approaches similar to [1-3]. They allow you to construct effective solutions even when the properties are described by complex functions.

\section{Statement of wear-contact problem}

Elastic pipe of the thickness $h_{\text {in }}$ and inner radius $r_{\text {in }}$ covered by thin nonuniform elastic layer of the thickness $h_{\text {out }}$ and inner radius $r_{\text {out }}$. Rigid cylindrical bush put on this coated tube with tension $\delta$ (see Figure 1). It is assumed that thickness $h_{\text {out }}$ of the coating is much smaller than bush length $2 a$ and inner radius $r_{\text {in }}$. Nonuniformity of coating means that elastic properties (Young's modulus $E_{\text {out }}(z)$ and Poisson's ratio $v_{\text {out }}(z)$ ) are functions of the coordinate $z$. It is also assumed that there is a smooth contact between layers and between bush outer layer along $z$ axis.
At time $\tau_{0}$ bush start to rotate around an axis $z$ with angular velocity $\omega$. Due to the presence of friction between the coating and the bush in the direction perpendicular to the axis $z$ (if $\omega$ is angular velocity vector with modulus $\omega$ and $\mathbf{r}$ is radius-vector of contact point than the friction direction is determined by the cross product $\boldsymbol{\omega} \times \mathbf{r}$ ), coating begins to wear out.

\subsection{Displacements due to load}

To derive the mathematical model of the problem, we replace bush by some distributed load $q(z, t)$ acting on region $z \in[-a, a]$ (under the bush), and equal to zero outside this region.

It can be shown that expressions for displacements of outer layer of tube with nonuniform coating due to load $q(z, t)$ almost coincide with expressions for the case of uniform layers (see, for example, $[4,5]$ ):

$$
\begin{aligned}
& u_{\mathrm{q}}(z, t)=-\frac{1-v_{\text {out }}^{2}(z)}{E_{\text {out }}(z)} h_{\text {out }} q(z, t) \\
& \quad-\frac{2\left(1-v_{\text {in }}^{2}\right)}{\pi E_{\text {in }}} \int_{-a}^{a} k_{\text {cyl }}\left(\frac{z-\zeta}{r_{\text {in }}}\right) q(\zeta, t) d \zeta .
\end{aligned}
$$

Here $E_{\text {in }}$ and $v_{\text {in }}$ are constant Young's modulus and Poisson's ratio of inner pipe, $k_{\mathrm{cyl}}(s)$ is known kernel of the cylindrical contact problem

$$
k_{\mathrm{cyl}}(s)=\int_{0}^{\infty} \frac{L(u)}{u} \cos (s u) d u,
$$

where

$$
L(u)=\frac{u\left[u^{2} B_{1}^{2}(u)-f(1, u) D_{1}^{2}(u)-1\right]}{S(u)},
$$




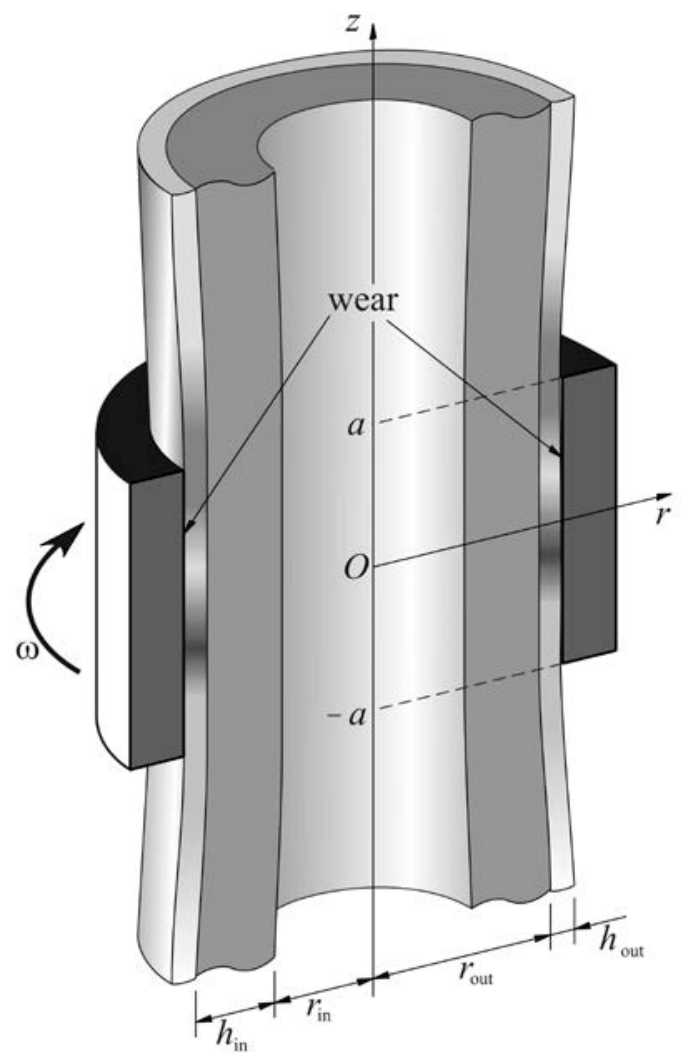

Fig. 1. Wear-contact cylindrical problem.

$$
\begin{gathered}
f(r, u)=\frac{2\left(1-v_{\text {in }}\right)}{r}+u^{2} r, k_{r}=\frac{r_{\text {out }}}{r_{\text {in }}}, \\
S(u)=\frac{f(1, u)}{k_{r}}+f\left(k_{r}, u\right)+k_{r} u^{4} A_{1}^{2}(u)-u^{2} f\left(k_{r} u\right) B_{1}^{2}(u) \\
-k_{r} u^{2} f(1, u) C_{1}^{2}(u)+f(1, u) f\left(k_{r}, u\right) D_{1}^{2}(u), \\
A_{1}(u)=\mathrm{I}_{0}(u) \mathrm{K}_{0}\left(k_{r} u\right)-\mathrm{I}_{0}\left(k_{r} u\right) \mathrm{K}_{0}(u), \\
B_{1}(u)=\mathrm{I}_{0}(u) \mathrm{K}_{1}\left(k_{r} u\right)+\mathrm{I}_{1}\left(k_{r} u\right) \mathrm{K}_{0}(u), \\
C_{1}(u)=\mathrm{I}_{0}\left(k_{r} u\right) \mathrm{K}_{1}(u)+\mathrm{I}_{1}(u) \mathrm{K}_{0}\left(k_{r} u\right), \\
D_{1}(u)=\mathrm{I}_{1}(u) \mathrm{K}_{1}\left(k_{r} u\right)-\mathrm{I}_{1}\left(k_{r} u\right) \mathrm{K}_{1}(u),
\end{gathered}
$$

and $\mathrm{I}_{0}(u), \mathrm{I}_{1}(u), \mathrm{K}_{0}(u), \mathrm{K}_{1}(u)$ are Bessel functions.

\subsection{Accounting of wear}

A lot of experiments shown that the wear velocity of body surface points is proportional to the normal load and relative speed of surfaces and inversely proportional to the material hardness [6-10]:

$$
v_{\mathrm{w}}(z, t)=-\frac{k_{\mathrm{w}} V q(z, t)}{H(z)},
$$

where $V=\omega\left(r_{\text {out }}+h_{\text {out }}\right)$ is speed of bush base points relative to the outer coating of points, $H(z)$ is hardness of coating, $q(z, t)$ is normal contact pressure, $k_{\mathrm{w}}$ is known constant.

Using (1) we can obtain that radial displacement of the outer face of coating due to wear has the form

$$
u_{\mathrm{w}}(z, t)=\int_{\tau_{0}}^{t} v_{\mathrm{w}}(z, \tau) d \tau=-\frac{k_{\mathrm{w}} V}{H(z)} \int_{\tau_{0}}^{t} q(z, \tau) d \tau .
$$

\subsection{Final integral equation}

According to equations (1) and (5) radial displacement of outer surface due to wear and applied load of coated tube has a form (we assume that wear $u_{\mathrm{w}}(z, t)$ is small compared to coating thickness $h_{\text {out }}$ )

$$
\begin{aligned}
u_{\text {out }}(z, t) & =-\frac{1-v_{\text {out }}^{2}(z)}{E_{\text {out }}(z)} h_{\text {out }} q(z, t)-\frac{k_{\mathrm{w}} V}{H(z)} \int_{\tau_{0}}^{t} q(z, \tau) d \tau \\
& -\frac{2\left(1-v_{\text {in }}^{2}\right)}{\pi E_{\text {in }}} \int_{-a}^{a} k_{\text {cyl }}\left(\frac{z-\zeta}{r_{\text {in }}}\right) q(\zeta, t) d \zeta .
\end{aligned}
$$

But displacement of the outer face of the tube under the bush equal to tension $\delta$ (we also assume that wear is small compare to tension) minus wear (because tension depend on time due to wear). Therefore the mixed integral equation for determining contact pressure $q(z, t)$ will have the following form

$$
\begin{aligned}
& \frac{1-v_{\text {out }}^{2}(z)}{E_{\text {out }}(z)} h_{\text {out }} q(z, t)+\frac{k_{\mathrm{w}} V}{H(z)} \int_{\tau_{0}}^{t} q(z, \tau) d \tau \\
& +\frac{2\left(1-v_{\text {in }}^{2}\right)}{\pi E_{\text {in }}} \int_{-a}^{a} k_{\text {cyl }}\left(\frac{z-\zeta}{r_{\text {in }}}\right) q(\zeta, t) d \zeta \\
& =\delta-\frac{k_{\mathrm{w}} V}{H(z)} \int_{\tau_{0}}^{t} q(z, \tau) d \tau .
\end{aligned}
$$

There exist several cases when the hardness of the materials is proportional to their contact rigidity (see, for example, $[11,12])$, i.e.

$$
H(z)=k_{\mathrm{H}} R(z),
$$

where $k_{\mathrm{H}}$ is known constant and $R(\mathrm{z})$ is contact rigidity of coating equal to

$$
R(z)=\frac{E_{\text {out }}(z)}{1-v_{\text {out }}^{2}(z)} .
$$

Earlier we used such assumptions in papers [2, 3]

According to equations (7) and (8) we obtain new mixed integral equation in following form

$$
\frac{h_{\text {out }}}{R(z)}(\mathbf{I}-\mathbf{V}) q(z, t)+\frac{2\left(1-v_{\text {in }}^{2}\right)}{\pi E_{\text {in }}} \mathbf{F} q(z, t)=\delta,
$$

where introduced indentity operator I, Volterra integral operator with constant kernel

$$
\mathbf{V} f(t)=-\int_{\tau_{0}}^{t} \frac{2 k_{\mathrm{w}} V}{k_{\mathrm{H}} h_{\text {out }}} f(\tau) d \tau,
$$

and Fredholm integral operator with symmetric positive difference kernels on finite interval:

$$
\mathbf{F} f(z)=\int_{-a}^{a} k_{\mathrm{cyl}}\left(\frac{z-\zeta}{r_{\mathrm{in}}}\right) f(\zeta) d \zeta .
$$

Note that function $R(z)$ describes elastic properties of coating. It can be rapidly changing or discontinuous.

The problem is to find the contact stresses $q(z, t)$ under the bush for its subsequent analysis.

\section{Mathematical model in dimensionless form}

Constructing a solution and studying the result is conveniently done in dimensionless variables. Therefore, 
we introduce the following dimensionless variables and functions

$$
\begin{gathered}
z^{*}=\frac{z}{a}, \quad \zeta^{*}=\frac{\zeta}{a}, \quad t^{*}=\frac{t}{\tau_{0}}, \quad \delta^{*}=\frac{\delta}{a}, \\
m^{*}\left(z^{*}\right)=\frac{h_{\text {out }} E_{\text {in }}}{2\left(1-v_{\text {in }}^{2}\right) a R(z)}, \\
q^{*}\left(z^{*}, t^{*}\right)=\frac{2\left(1-v_{\text {in }}^{2}\right) q(z, t)}{E_{\text {in }}}, \\
K^{*}\left(t^{*}, \tau^{*}\right)=-\frac{2 k_{\mathrm{w}} V \tau_{0}}{k_{\mathrm{H}} h_{\text {out }}}=-V^{*}=\text { const, } \\
k^{*}\left(z^{*}, \zeta^{*}\right)=\frac{1}{\pi} k_{\text {cyl }}\left(\frac{z-\zeta}{r_{\text {in }}}\right), \\
\mathbf{V}^{*} f\left(t^{*}\right)=\int_{1}^{t} K\left(t^{*}, \tau^{*}\right) f\left(\tau^{*}\right) d \tau^{*}, \\
\mathbf{F}^{*} f\left(z^{*}\right)=\int_{-1}^{1} k^{*}\left(z^{*}, \zeta^{*}\right) f\left(\zeta^{*}\right) d \zeta^{*} .
\end{gathered}
$$

Then mathematical model for the problem in dimensionless form is mixed integral equation

$$
m^{*}\left(z^{*}\right)\left(\mathbf{I}-\mathbf{V}^{*}\right) q^{*}\left(z^{*}, t^{*}\right)+\mathbf{F}^{*} q^{*}\left(z^{*}, t^{*}\right)=\delta^{*} .
$$

We should find function $q^{*}\left(z^{*}, t^{*}\right)$ (and hence the contact stresses $q(z, t))$ from this equation.

\section{Solution of the problem}

It is required to find function $q^{*}\left(z^{*}, t^{*}\right)$ from equation (14). Note that this equation contain integral operators of different types and function $m^{*}\left(z^{*}\right)$ connected with coating nonuniformity, i.e. it can be rapidly changing or even discontinuous.

If we will use usual representations for the function $q^{*}\left(z^{*}, t^{*}\right)$ like

$$
q^{*}\left(z^{*}, t^{*}\right)=\sum_{i=0}^{\infty} f_{i}\left(t^{*}\right) p_{i}\left(z^{*}\right)
$$

where $\left\{p_{i}\left(z^{*}\right)\right\}$ is standard basis $\left(p_{i}\left(z^{*}\right)\right.$ are polynomials, trigonometric functions, for example), than it will lead us to computational errors because such a solution representation and system of basis functions does not use information about complex function $m^{*}\left(z^{*}\right)$.

But if we will use this information we will obtain effective solution. The construction of the solution of a similar equation taking into account a complex function was carried out in [13]. So we can write out final solution of this equation in following form

$$
q^{*}\left(z^{*}, t^{*}\right)=\frac{1}{m^{*}\left(z^{*}\right)} \sum_{k=0}^{\infty} f_{k}\left(t^{*}\right) \varphi_{k}\left(z^{*}\right),
$$

where

$$
\begin{gathered}
f_{k}\left(t^{*}\right)=\left(\mathbf{I}+\mathbf{W}_{k}\right) \frac{\delta^{*} \sqrt{J_{0}} \psi_{0}^{(k)}}{1+\gamma_{k}}, \\
\varphi_{k}\left(z^{*}\right)=\sum_{i=0}^{\infty} \psi_{i}^{(k)} p_{i}\left(z^{*}\right), \\
\mathbf{W}_{k} f\left(t^{*}\right)=\int_{1}^{t} R_{k}\left(t^{*}, \tau^{*}\right) f\left(\tau^{*}\right) d \tau^{*},
\end{gathered}
$$

$$
\begin{gathered}
J_{i}=\int_{-1}^{1} \frac{\left(\zeta^{*}\right)^{i}}{\sqrt{m^{*}\left(\zeta^{*}\right)}} d \zeta^{*}, \quad p_{0}\left(z^{*}\right)=\frac{1}{\sqrt{J_{0}}}, \\
p_{j}\left(z^{*}\right)=\frac{1}{\sqrt{d_{j-1} d_{j}}}\left|\begin{array}{cccc}
J_{0} & J_{1} & \cdots & J_{j} \\
J_{1} & J_{2} & \cdots & J_{j+1} \\
\vdots & \vdots & \ddots & \vdots \\
1 & z^{*} & \cdots & \left(z^{*}\right)^{j}
\end{array}\right|, \\
d_{i}=\left|\begin{array}{cccc}
J_{0} & J_{1} & \cdots & J_{i} \\
J_{1} & J_{2} & \cdots & J_{i+1} \\
\vdots & \vdots & \ddots & \vdots \\
J_{i} & J_{i+1} & \cdots & J_{2 i}
\end{array}\right|,
\end{gathered}
$$

coefficients $\psi_{i}^{(k)}$ and $\gamma_{k}$ can be find from the solution of spectral problem

$$
\sum_{l=0}^{\infty} R_{i l} \psi_{l}^{(k)}=\gamma_{k} \psi_{i}^{(k)}, \quad i, k=0,1,2, \ldots,
$$

$R_{i l}$ can be calculated from the formula

$$
R_{i l}=\int_{-1-1}^{1} \int_{-1}^{1} \frac{k^{*}\left(z^{*}, \zeta^{*}\right) p_{i}\left(z^{*}\right) p_{l}\left(\zeta^{*}\right)}{m^{*}\left(z^{*}\right) m^{*}\left(\zeta^{*}\right)} d z^{*} d \zeta^{*},
$$

and $R_{k}\left(t^{*}, \tau^{*}\right)$ are resolvents of the kernels

$$
K_{k}\left(t^{*}, \tau^{*}\right)=\frac{K^{*}\left(t^{*}, \tau^{*}\right)}{1+\gamma_{k}}=-\frac{V^{*}}{1+\gamma_{k}} .
$$

Since the kernel $K^{*}\left(t^{*}, \tau^{*}\right)$ is constantly then kernels $K_{k}\left(t^{*}, \tau^{*}\right)$ are also constantly. Then their resolvents can be calculated by the formula [14]

$$
R_{k}\left(t^{*}, \tau^{*}\right)=-\frac{V}{1+\gamma_{k}} \exp \left[-\frac{V^{*}\left(t^{*}-\tau^{*}\right)}{1+\gamma_{k}}\right] .
$$

According to formula (21) representation (17) for functions $f_{k}(t)$ will take a form

$$
f_{k}\left(t^{*}\right)=\frac{\delta^{*} \sqrt{J_{0}} \psi_{0}^{(k)}}{1+\gamma_{k}} \exp \left[-\frac{V^{*}\left(t^{*}-1\right)}{1+\gamma_{k}}\right] .
$$

These functions decreases over time. According to (16), (17), and (22) (here $z^{*} \in[-1,1], t^{*} \geq 1$ )

$$
\begin{aligned}
& q^{*}\left(z^{*}, t^{*}\right) \\
& =\frac{\delta^{*} \sqrt{J_{0}}}{m^{*}\left(z^{*}\right)} \sum_{k=0}^{\infty} \frac{\psi_{0}^{(k)}}{1+\gamma_{k}} \exp \left[-\frac{V^{*}\left(t^{*}-1\right)}{1+\gamma_{k}}\right] \sum_{i=0}^{\infty} \psi_{i}^{(k)} p_{i}\left(z^{*}\right) .
\end{aligned}
$$

The expression for the contact pressure in the dimensional form is $\left(z \in[-a, a], t \geq \tau_{0}\right)$

$$
\begin{aligned}
& q(z, t)=\frac{\delta \sqrt{J_{0}} R(z)}{h_{\text {out }}} \\
& \times \sum_{k=0}^{\infty} \frac{\psi_{0}^{(k)}}{1+\gamma_{k}} \exp \left[-\frac{2 k_{\mathrm{w}} V\left(t-\tau_{0}\right)}{k_{\mathrm{H}} h_{\text {out }}\left(1+\gamma_{k}\right)}\right] \sum_{i=0}^{\infty} \psi_{i}^{(k)} p_{i}\left(\frac{z}{a}\right) .
\end{aligned}
$$

Consequently, contact stresses will be negligible over time. This is because the material under the bush wears out. Over time, the tension should disappear.

But we can use this solution only for case when wear is significantly less than coating thickness $h_{\text {out }}$. Otherwise, the integral equation would become nonlinear

$$
\frac{1-v_{\text {out }}^{2}(z)}{E_{\text {out }}(z)}\left(h_{\text {out }}-\frac{k_{\mathrm{w}} V}{H(z)} \int_{\tau_{0}}^{t} q(z, \tau) d \tau\right) q(z, t)
$$




$$
\begin{aligned}
& +\frac{k_{\mathrm{w}} V}{H(z)} \int_{\tau_{0}}^{t} q(z, \tau) d \tau \\
& +\frac{2\left(1-v_{\text {in }}^{2}\right)}{\pi E_{\text {in }}} \int_{-a}^{a} k_{\text {cyl }}\left(\frac{z-\zeta}{r_{\text {in }}}\right) q(\zeta, t) d \zeta \\
& =\delta-\frac{k_{\mathrm{w}} V}{H(z)} \int_{\tau_{0}}^{t} q(z, \tau) d \tau,
\end{aligned}
$$

The process of finding solution of (26) would be much more complicated. Our solution can be first step in numerical or analytical algorithm for finding solution of this nonlinear problem. Moreover, sometimes it is important to use information about the coating production process. In this case, one can use the theory developed, for example, in [15].

Note that it is possible to find solution if tension $\delta$ is unknown. It is possible if we know some additional information, compression force, for example. In this case we obtain mixed integral equation with partially unknown right side and system of additional equations and should use special projection method [16] for finding solution of such a problem.

\section{Conclusions}

Cylindrical contact-wear problem for rigid bush and elastic tube with complex coating is posed and solved. Analytical representation for contact stresses under the bush is presented in series over special system of basis functions. This system depends on initial data in main integral equation. It is shown that contact stresses will be negligible over time.

Contact stiffness $R(z)$ of the coating is represented by separate factor in final expression for contact stresses. Due to this fact, the solution obtained allows using a small number of members of the series to obtain a reliable result. It allows one to perform effective numerical computations for any types of coating nonuniformities (even discontinuous). Another approaches does not provide this possibility because they use expansions on standard basis functions (Legendre polynomials, trigonometric functions, etc.).

It is noted that the solution obtained is valid for small wear compared coating thickness. Otherwise we should use nonlinear mathematical model (25). It is complicated problem, but obtained solution can be first step in numerical or analytical algorithm for finding its solution.
The work was partially supported by the Russian Foundation for Basic Research (projects Nos. 18-01-00770 and 18-0100920).

\section{References}

1. A.V. Manzhirov, K.E. Kazakov, Dokl. Phys. 62 (7), 344-349 (2017) DOI 10.1134/S1028335817070035

2. A.V. Manzhirov, K.E. Kazakov, AIP Conf. Proc. 1959, 070023 (2018) DOI 10.1063/1.5034698

3. K.E. Kazakov, IOP Conf. Ser.: Mater. Sci. Engng 489, 012027 (2019) DOI 10.1088/1757-899X/489/1/ 012027

4. A.V. Manzhirov, V.A. Chernysh, Mech. Solids 22(6), (1988)

5. N.Kh. Arutyunyan, A.V. Manzhirov, Contact problems in the theory of creep (Izd-vo Inst. Mekhaniki NAN Armenii, Yerevan, 1999) [in Russian]

6. A.S. Pronikov, Wear and durability of machines (Mashgiz, Moscow, 1957) [in Russian]

7. M.M. Khrushchev, M.A. Babichev, Abrasive wear (Nauka, Moscow, 1970) [in Russian]

8. J. Collins, Failure of materials in mechanical design: analysis, prediction, prevention (Wiley, New York, 1993)

9. I.G. Goryacheva, M.N. Dobychin, Contact problems in tribology (Mashinostroenie, Moscow, 1988) [in Russian]

10. I.A. Soldatenkov, Wear contact problem with applications to engineering calculation of wear (Fizmatkniga, Moscow, 2010) [in Russian]

11. J.F. Archard, J. Appl. Phys. 24 (8), 981-988 (1953) DOI 10.1063/1.1721448

12. A. Schalliamach, Proc. Phys. Soc.. Sect. B 67 (12), 883-891 (1954) DOI 10.1088/0370-1301/67/12/304

13. A.V. Manzhirov, K.E. Kazakov, Lect. Notes Engng Comp. Sci. 2224, 877-882 (2016)

14. A.D. Polyanin, A.V. Manzhirov, Handbook of integral equations, 2nd edition (Chapman \& Hall/ CRC, Boca Raton, 2008)

15. D.A. Parshin, J. Phys.: Conf. Ser. 1203, 012006 (2019) DOI 10.1088/1742-6596/1203/1/012006

16. A.V. Manzhirov, Dokl. Phys. 61 (10), 489-493 (2016) DOI 10.1134/S1028335816100025 